\title{
The road to regionalization in congenital heart surgery: a narrative review
}

\author{
Hiba Z. Ghandour ${ }^{1 \wedge}$, Karl Welke², Tara Karamlou ${ }^{3}$ \\ ${ }^{1}$ Department of Thoracic and Cardiovascular Surgery, Heart, Vascular, and Thoracic Institute, Cleveland Clinic, Cleveland, OH, USA; ${ }^{2}$ Division \\ of Congenital Cardiothoracic Surgery, Atrium Health Levine Children's Hospital, Charlotte, NC, USA; ${ }^{3}$ Department of Pediatric Cardiac Surgery, \\ Heart, Vascular, and Thoracic Institute, Cleveland Clinic, Cleveland, OH, USA \\ Contributions: (I) Conception and design: All authors; (II) Administrative support: None; (III) Provision of study materials or patients: All authors; \\ (IV) Collection and assembly of data: All authors; (V) Data analysis and interpretation: All authors; (VI) Manuscript writing: All authors; (VII) Final \\ approval of manuscript: All authors. \\ Correspondence to: Tara Karamlou, MD, MSc. Professor of Surgery, Department of Pediatric Cardiac Surgery, Cleveland Clinic Children's Hospital, \\ M-41-022A, 9500 Euclid Avenue, Cleveland, OH 44195, USA. Email: karamlt@ccf.org.
}

Objective: This review article will highlight the current state of pediatric cardiac surgical care delivery in the United States (U.S.), examine the data that supports regionalization along with the potential drawbacks of such a system, specifically with regard to how these efforts might impact socioeconomic status (SES) and racial/ethnic disparities. We will also discuss global implications for regionalization, and whether emerging healthcare delivery systems should be based on this concept.

Background: Concentrating pediatric cardiac surgical care to regional high-volume specialty centers would likely improve patient survival and promote more efficient use of resources. Recent initiatives in two states have proposed minimum volume requirements for pediatric cardiac surgery programs. However, implemented poorly, such a change could exacerbate accessibility issues and would face obstacles in the U.S., including regulatory constraints, antitrust actions and balancing of financial incentives. Real-world studies, as well as theoretical models, indicate that patients undergoing pediatric cardiac surgery at high-quality centers with larger volumes fare better. Other paradigms for care delivery exist across the U.S., including healthcare satellite systems and tiered systems. These alternative models that consist of a large hospital that participates in specialty care for neighboring associated small hospitals or designate certain centers (usually higher tiered centers) to provide a cache of services. Both models usually require financial alignment, geographic proximity among the participants, and extensive care coordination that defines thresholds for referral of care to the larger hospital.

Methods: A comprehensive narrative review on congenital heart surgery (CHS), regionalization, and healthcare policy efforts was carried out. PubMed was used to search articles published on the topic in the past 20 years. The search yielded 944 studies, of which 38 satisfied the inclusion criteria for narrative review.

Conclusions: There are no easy answers to the complex questions of whether and how pediatric cardiac surgery should be regionalized to specialty centers in the U.S. or on an international level. Nevertheless, although evidence supports centralization to save lives and lower costs, implementation is challenged by financial and social reasons. Existing centralized services, as exist for organ transplantation and transcatheter valve replacement, demonstrate that regionalization makes sense for complex interventions.

Keywords: Regionalization; centralization; congenital heart surgery (CHS); healthcare policy

\footnotetext{
$\wedge$ ORCID: 0000-0002-3375-6600.
} 
Received: 20 July 2021; Accepted: 22 March 2022; Published: 30 September 2022.

doi: $10.21037 /$ asj-21-70

View this article at: https://dx.doi.org/10.21037/asj-21-70

\section{Background}

The complex nature of congenital heart surgery (CHS) necessitates a collaborative and devoted multi-disciplinary team to optimize outcomes. The positive association between higher surgeon and center volumes and lower 30-day mortality, morbidity, length of stay, and cost for infants undergoing open heart surgery has been established. Regionalization can operationalize this relationship to improve outcomes (1). Regionalization allows the number of hospitals offering specialized care to be condensed to those meeting an established quality metric (volume is one of these that is easy to measure and therefore frequently utilized), while overcoming geographical hurdles.

Regionalization may carry potential downsides, though some of these are hypothetical as the real impact is unknown (2). Purported concerns include: (I) unequal access that could magnify healthcare inequities and reduce access to vulnerable socioeconomic status (SES)/racial/ ethnic groups; (II) reduction in hospital numbers, creating a monopoly effect; (III) educational and subspecialty service ramifications with loss of pediatric cardiac surgical services; (IV) risk aversion and diversion. Further research is warranted, some of which is currently underway.

The aim of this review is to consolidate financial, access, ethical and international lessons learned from past efforts aimed at regionalization, characterize knowledge gaps, and suggest possible next steps needed to pave the 'road to regionalization'.

We present the following article in accordance with the Narrative Review reporting checklist (available at https:// asj.amegroups.com/article/view/10.21037/asj-21-70/rc).

\section{Methods}

PubMed was used to search articles pertaining to CHS, regionalization, financing infant heart surgery, ethical challenges, as well as international implications published in the last 20 years. To ensure a comprehensive review, our search strategy combined 'congenital heart surgery' AND 'regionalization' OR 'centralization' AND 'healthcare policy'. Inclusion criteria included a manuscript (commentary, editorial, systematic review, meta- analysis, original article, review, book chapter, government publication, legislative documents) published in English between 1/1/2001 and 08/31/2021. Exclusion criteria included articles published outside the time period listed, or publications within the time period that focused on other pediatric surgical sub-specialties, regionalization in stroke and other surgical sub-specialties and healthcare policy unrelated to congenital heart disease (CHD). Our NCBI, PubMed search yielded 944 studies. Of these, 38 articles met the inclusion criteria and were thus used in our paper.

\section{CHS financing}

Ensuring optimal quality in the delivery of CHS is a path of its own, lest the road of adequately financing it. Fiscal constraints have the potential to negatively impact the quality of care in finance-driven organizations by creating practices that reduce costs but limit resources and personnel over time. It is unclear whether these 'scale-down' practices will worsen benchmarked outcomes.

Avenues to overcome fiscal constraints may be found in several hospital financial determinants, which have the power to significantly influence quality of care in cardiovascular disease. Factors such as hospital size, asset liquidity, labor costs, operating efficiency and capital structure play a vital role in the quality of care offered to patients (3). A first-difference regression has suggested that hospitals with more profit and debt financing, will generally have improved quality of care (3). More profit implies a strong ability to train the workforce, hire a skilled nursing team, decrease patient wait times, ameliorate safety concerns and purchase novel medical equipment.

These findings contribute to the cost-effectiveness/ cost-savings argument between administrating one big hospital versus multiple small centers. Our group's work in simulating regionalization models using different volume and population thresholds $(1,4)$, as well as prior work from Welke et al. (4) suggests that higher annual case volumes are associated with lower mortality, though whether volume is the best surrogate for quality of care delivered remains unclear. Although hospital costs were not assessed in these studies, reduction in hospital costs have been associated with adult congenital care delivery 
by dedicated congenital cardiac surgeons compared to adult acquired surgeons (1). These data, in concert with the financial argument posed earlier, suggest that downsizing to a limited number of high volume centers would create a positive feedback cycle between improved quality and cost reduction. In point of fact, one definition of valuebased care includes morbidity and mortality divided by cost, such that reductions in mortality and morbidity must come at a reasonable financial investment. These lessons can be applied to the globalization equation as value-based care propositions leveraged in the United States (U.S.) can be used to inform CHS regionalization efforts in countries with extensive hospital systems, and those in earlier phases of centralization.

\section{Healthcare disparities: access challenges augmented or mitigated?}

Disparities occur in the access and provision of healthcare across racial, ethnic, and socioeconomic groups. The U.S. Office of Disease Prevention and Health Promotion describes disadvantaged populations as groups of people who have experienced greater obstacles to health based on their racial or ethnic group, religion, socioeconomic status (SES), gender, age, occupation, mental health, cognitive, sensory or physical disability or other characteristics linked to discrimination or exclusion (5). How can these disparities impact CHS outcomes and what should regionalization efforts focus on to mitigate them?

Previous investigations have demonstrated associations between both race/ethnicity and SES outcomes after CHS; however, the underlying reasons for the discrepancies are unclear (6-9). Some disparities are attributable to the differences in the hospitals where the patients receive care. Black patients and those who are socioeconomically disadvantaged tend to be cared for at lower quality hospitals (10-12). The systems of care for CHD patients differ from care for adults due to the relative infrequency of CHS and the resource intensity needed to care for this population. However, there is wide variation in mortality and other outcomes across hospitals, possibly creating qualitybased inequities. The degree to which race/ethnicity and SES factors influence access to high quality hospitals for CHS patients has not been established. Also unknown is whether SES and race/ethnicity influence the type of care provided or the degree to which these factors complicate discharge planning or change the availability of surveillance mechanisms (such as home monitoring programs) for single ventricle patients. A variable racial/ethnic distribution is observed for the CHD population, particularly the population requiring CHS, and this may impact access to high-quality medical services and the equitable provision of state and federal services.

A recent investigation by Karamlou et al. (13) showed that both race/ethnicity and income level are important factors that impact in-hospital mortality, with African American and mixed-race children having the highest death rates. The latter may be critical insofar as mixedrace families have unique psychological and environmental stressors that lead to higher rates of poor birth outcomes. We have yet to investigate how different regionalized systems might impact healthcare disparities, which is often raised as a critical obstacle to realizing equitable benefits from centralized care. Important differences exist between patients undergoing CHS and those admitted for other reasons. Surgical patients presented at an older age and had higher median income levels compared to those having non-interventional care. While we are not yet able to identify the reasons for these differences, we suspect it may be due to differences in thresholds for referral for the more prevalent 'elective' cases, access to care, or the robustness of longitudinal surveillance. Further investigation should focus on identifying specific points along the CHD care continuum (especially with the context of the type of therapy required) where disparities associated with poor outcome are magnified.

Another critical avenue of investigation is whether processes of care that could be impacted by regionalization modify existing interactions between these and SES and racial/ethnic factors. Karamlou et al. studied 166,599 CHD admissions from 52 hospitals, of which 58,395 underwent CHS and assessed whether interactions between SES and other patient and hospital factors could mitigate or potentiate adverse outcomes among this national cohort of patients both with surgical and non-surgical CHD (13). For example, was there an income level at which the impact of ethnicity was 'neutralized', or did race/ethnicity or low income disproportionately impact the outcome of more severe forms of CHD, such as hypoplastic left heart syndrome? The authors found that the relationship between median income and mortality is parabolic rather than linear, as previous studies have described. Patients of both lower median income and higher median income fared worse than patients with median income between $\$ 72,000$ and $\$ 80,000$ (Figure 1). While the relative disadvantage imparted by higher income may seem counterintuitive, the 


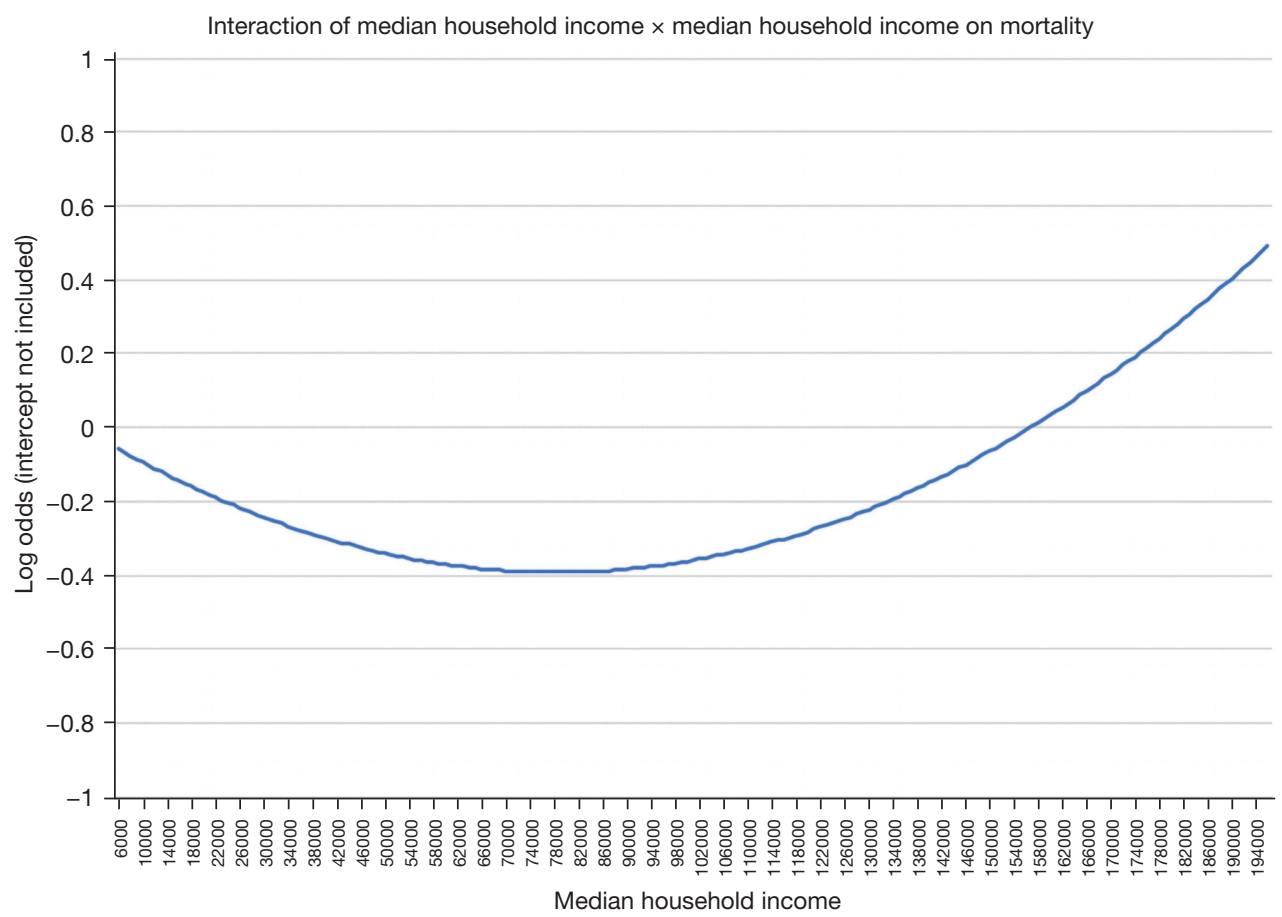

Figure 1 The relationship between median household income (horizontal axis) and the probability of death (vertical axis) over the range of available data was non-linear, with higher risk at lower and higher income levels. The risk of death nadirs between annual income of $\$ 72,000-\$ 80,000$.

authors hypothesized that higher income patients, who are financially equipped to travel, might be accessing more complex care that is delivered only at regionalized centers (i.e., transplant, unifocalization of major aortopulmonary collateral arteries). This hypothesis, however, is speculative and requires further study in future research.

An added research focus is whether, should regionalization efforts be actualized, current inpatient processes should differ among specific fragile SES or racial/ ethnic groups. The study by Karamlou et al. (13) found that hospital length of stay among Black neonates was longer than other racial and ethnic groups because specific interventions such as permanent feeding tubes (as compared to temporary feeding tubes) and tracheostomy were deployed with increased prevalence. Although intent could not be inferred from this retrospective study, it is possible that length of stay was prolonged because providers wanted a more robust 'safety-net' during the outpatient follow-up period. As to the interaction between race/ethnicity and SES in regionalization of CHD care in the context of the volume-outcomes relationship, we speculate that larger volume hospitals may perform better compared to smaller volume hospitals. The median total annual admission volume over our study period was 2,812 (IQR: 1,740-3,839); however, hospital population varied widely, with racial/ ethnic composition and median neighborhood household income varying by hospital (13). Alternatively, if the smaller volume hospital is in a geographically-diverse region and has evolved safety nets to resolve healthcare disparities, volume may no longer be an important determinant of outcomes among the socially disadvantaged or less prevalent ethnicities (13). Further studies are needed to delineate whether modified care pathways have a causative relationship between prolonged length of stay and reduced mortality among black neonates. Additional population health initiatives and targeted resource allocation are needed to increase access for the vulnerable, disadvantaged racial and SES population to high-quality, multidisciplinary programs. Additional studies to decrease the healthcare access gap among the under-resourced, socially challenged, non-English speaking populations are warranted. Of note, a study by Woo et al. showed that infants with severe CHD who are publicly insured may need to increase their travel distance if they are restricted to seek care in-state solely; 


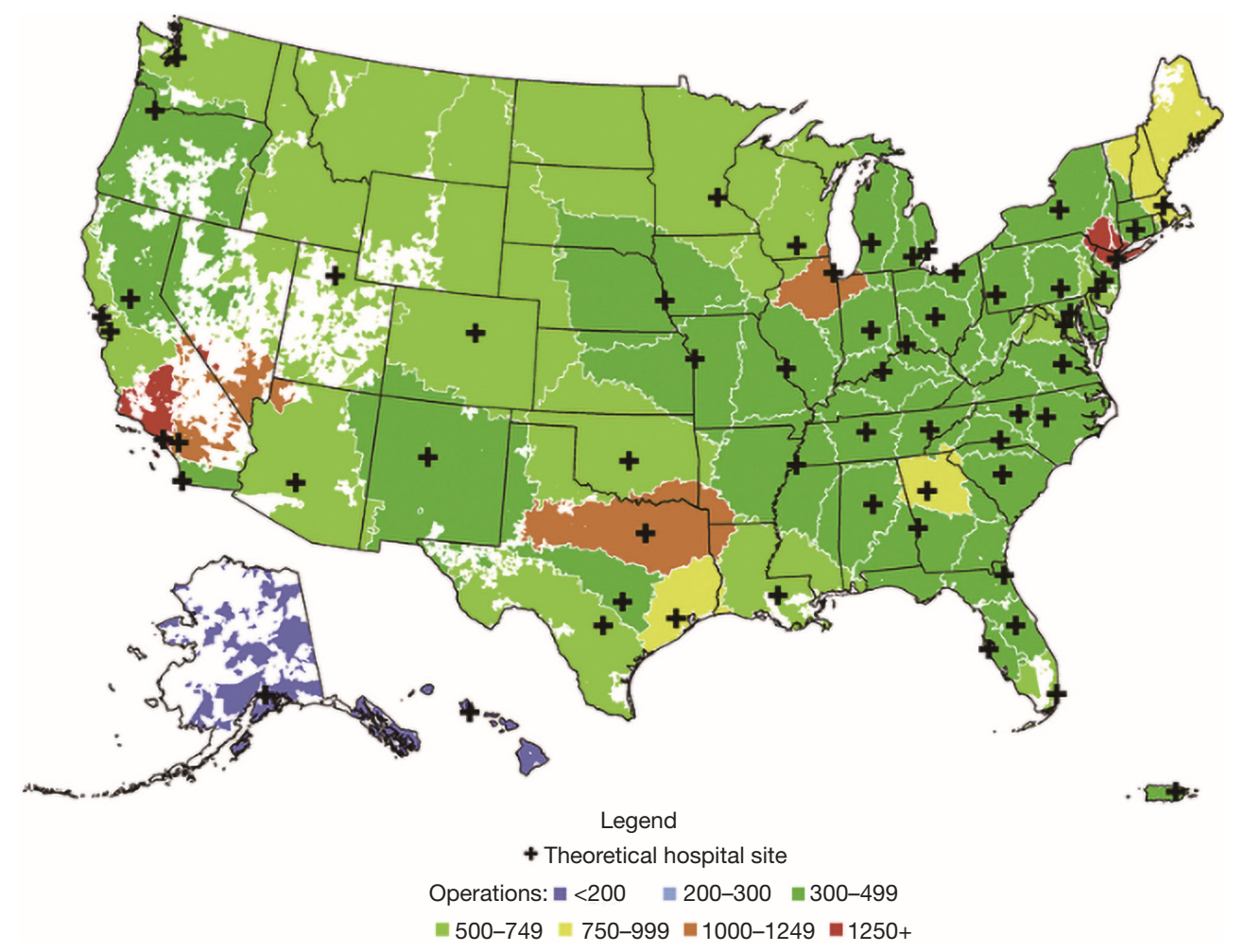

Figure 2 Map of the U.S. showing the locations of theoretical hospitals following regionalization. Each hospital, except those in AK and HI, would have an annual volume of 300 or more operations. With all patients being treated at their closest hospital, national mean and median travel distances would be reduced by $34 \%$ and $9 \%$ respectively over the present environment.

in other words, the study highlighted that receiving outof-state care may mitigate access barriers to some and thus future regionalization efforts should take into consideration decreasing these access barriers particularly for the publicly insured infants (14).

There are no clear answers as to how regionalization might impact fragile SES and racial/ethnic minorities. However, our data regarding travel incurred among patients and families in a regionalized CHS system is reassuring. Relying on existing hospitals, we demonstrated that with regionalization of CHS to hospitals performing 300 or more operations annually, there would be a modest increase in median travel distance from 38.5 to 69.6 miles (15). The differential impact of this increase on patients of different SES and racial/ethnic groups was not elucidated.

Beginning with a theoretical blank slate and using objective methodology guided by population density and volume thresholds to estimate the optimal number and locations of hospitals needed to provide CHS to patients in the U.S. we found more promising results (4). We created a novel algorithm to determine the locations of potential hospitals and the geographic areas and the numbers of patients they would serve, then calculated the theoretical travel distances for patients in this idealized system.

We theorized that the U.S. could be served by $60 \mathrm{CHS}$ hospitals, at least a $56 \%$ reduction over the current number (Figure 2). Each hospital, except those in AK and HI, would have an annual volume of 300 or more operations. With all patients being treated at their closest hospital, national mean and median travel distances would be reduced by $34 \%$ and $9 \%$ respectively over the present environment. This study described an "ideal" that future regionalization frameworks may be referenced against. While these are simulated data that did not include variables linked to either SES status not race or ethnicity, a reduction in travel distance for all would likely benefit patients of all strata. Further studies that will inform paradigms for regionalization that minimize healthcare disparities among CHS patients and provide the largest survival advantage while minimizing imposed hardships are clearly needed.

The recent COVID-19 pandemic has highlighted the utility of virtual health platforms, and telemedicine. While 
not a substitute for surgery, telehealth services can extend specialty care, assist with triage and improve continuity of care for patients in remote areas. Further data regarding the sustainability of telemedicine is needed.

\section{The ethical dilemma}

The road to regionalization necessitates the scaffolding of the four principles of medical ethics: autonomy, beneficence, non-maleficence and justice. Tools used to ensure the principles are respected are part and parcel of the healthcare system and include informed consent, which is an ethical and legal document detailing details of a procedure, risks associated with the procedure, as well as benefits, and alternatives (16). When discussing procedures as complex as CHS, in addition to informed consent, shared decision making between patients and their surgical providers is a cornerstone to identifying the best therapeutic modality suited for the respective case.

Consequentially, when a patient presents with their parents, or their proxy decision makers, cardiologists and surgeons have an obligation to discuss the various treatment modalities available for the patient, as well as risks, including morbidity and mortality, benefits, and alternatives (17). When morbidity and mortality are discussed with parents, highlighting a center's own rates is important, even if their low case volumes have led to high morbidity and mortality. Despite this sounding as counter-intuitive to a center's profit and reputation, it is important to ensure complete transparency and respect to the process of informed consent. Parents have the right to choose, thereon, which center they would like to seek care in with the best interest of their child in mind. Informed consent cannot be complete if parents are unaware of the risks different centers may pose on the surgical outcomes of their child.

In the process of regionalization, patients and their well-being should always precede personal and corporate interests; this can only be possible when full transparency, informed consent and shared decision making are made viable for parents. Additional information aimed at guiding informed decision making in cardiac surgical centers includes public reporting. While some data on overall operative and adjusted operative mortality is available on the current STS-CHSD website at https://publicreporting. sts.org/chsd-exp, more user friendly information is needed. Parents and patients should be encouraged to review center specific, as well as surgeon specific outcomes to make an informed decision on where to seek care.

\section{International state of CHS}

The international arena of CHS regionalization is yet to be established. Given the significant gaps surrounding the availability of services and the varying factors influencing CHD outcomes in low-resource settings, it is helpful to assess the international landscape with a 30,000-foot view. Factors such as lack of cardiac surgery centers in several low- and middle-income countries, high cost of care and concomitant lack of insurance schemes capable of covering CHD repair services, lead to important hardships for families and patients (18). These factors contribute to late case presentations; patients additionally present with comorbid conditions, making them particularly challenging to local teams not equipped with the knowledge and techniques to address them. Combined with limited resources and understaffing in existing centers (18), ensuring optimal outcomes in such low-resource settings is severely challenged.

In one study on CHD neonatal outcomes in Western India between 2009 and 2011, one significant predictor of mortality was incomplete repair of CHD lesions (19). Other challenges discussed by the authors were late presentation of patients, with only $5 \%$ diagnosed in the antenatal stage (19). Interestingly, mode of transportation, i.e., private versus transport ambulance did not impact mortality (19). Furthermore, the total burden of CHS in India remains speculative, given the lack of national registries and an urgent need for them (20).

Other challenges facing CHS in low-resource settings include prolonged waiting time until surgery and paucity of human resources. In Thailand, median waiting time for pediatric patients referred for cardiac surgery was 195 days, with a $5 \%$ mortality at 2 years while waiting (21). Such phenomena are a threat to public health and attempts to resolve such challenges should ideally be made at the national level, in collaboration with international societies and experts. The paucity of human resources was highlighted in a survey of cardiac surgeons and residents in Nigeria, which showed the daunting need for pediatric cardiac surgery centers in the setting of a weak national health system, and poor human resources. Respondents emphasized the need for local and international partnerships with country leadership in hopes of advancing care for the pediatric population (22). Such barriers emphasize the urgent need to focus attention on low-resource settings, to ensure timely and high quality access to CHS services.

Both the wealth of a nation and the size of its population 
contribute to the ability to provide specialized care. Five out of 58 small, developing island states, have only local health centers, which are dependent on and served by visiting surgeons. Aruba has no local cardiac surgery center, necessitating the state to send its cardiac patients abroad, costing it $12 \%$ of its total healthcare expenditure (23). While itinerate surgeons provide a needed service, the model is unable to fill the needs of these nations.

Access to treatment for CHD in sub-Saharan Africa within 2 years of birth is $<1 \%$ (24). Nearly 300,486 infants are born annually in Africa with CHD (25). Even though surgical palliation of functionally single ventricle patients can improve survival, children with these severe lesions have very poor care coverage in the African region because such care is expensive and therefore is not highly prioritized by policymakers from a cost-effectiveness perspective $(26,27)$. Thus, ongoing education, collaboration with international societies, local civil society and policy makers is vital if progress is access to CHD surgical services is to be increased.

The global challenges discussed above demonstrate an urgent need for international collaboration between local CHD physicians, economic and technical experts to establish and/or advance access to CHS services within many burgeoning healthcare systems. The proposed structures, which should include centralization, will be variable and should be tailored to the needs of every country based on its national health system, population demographics, infrastructure, and insurance schemes used.

\section{International implications of regionalization}

In 2015, the landmark United Nations resolution "Transforming Our World: the 2030 Agenda for Sustainable Development" galvanized the global adoption of Universal Health Coverage by 2030. This resolution emphasized access to quality essential healthcare services, medications, and vaccines for all. In the same year, the World Health Organization recognized surgical and anesthesia care as an essential component of universal health coverage (Resolution WHA68.15) (28). These goals and resolutions are in stark contrast to the current reality. In low- and middle-income countries, over $90 \%$ of patients with CHD lack access to the care they need, resulting in nearly 200,000 excess child deaths each year (29,30). Furthermore, recent data highlights that high-income countries are served by 9.51 pediatric cardiac surgeons per million pediatric population, whereas low-income countries have 0.07 per million pediatric population (31). Asia and sub-Saharan Africa have one cardiac center per 16 million and 33 million people, respectively (32).

Minimizing variation in healthcare delivery and improving access to high quality care through regionalization has been proposed in high-income countries. Regionalization efforts in high-income countries have been carried out, with Sweden being among the first to show a reduction in overall 30-day mortality for infants undergoing open heart surgery from $9.5 \%$ to $1.9 \%$ post-regionalization (33). The centralization efforts in Sweden have resulted in longer travel distances for patients and their families, necessitating air transport more frequently; however, no increased risks of air transport were delineated compared to ambulance transport (33). Centralization increased the annual number of pediatric heart operations from 550 to 620; of these, the percentage of open heart procedures increased from $60 \%$ to $79 \%$ (33). Furthermore, these efforts improved collaboration and communication amongst local hospitals and the two centralized surgical centers. Our team highlighted the value and impact that regionalization could potentially have on patients' outcomes in the U.S. Our theoretical model for CHS delivery suggests that the U.S. can be served by almost 100 fewer centers than what currently exists, with a decrease in median travel distance from 38.5 to 35.1 miles, and a theoretical median hospital volume threshold of 451 annual operations, augmenting favorable outcomes (4). Both the Sweden and U.S. examples highlight the positive volumeoutcomes relationship; it should be noted nonetheless that these two countries have significant differences in their geography, number of centers and healthcare coverage infrastructure, making a head-on comparison imperfect.

An additional example of regionalization in a highincome country includes the Japan experience, which has demonstrated promise in the arena of regionalization. In its 2015-2016 status of cardiac surgery, the Japanese cardiovascular surgery database demonstrated that complex procedures, such as the Norwood, Rastelli and bidirectional Glenn were solely taking place at high volume centers. Despite this fact, complication rates, such as unplanned cardiac reoperation and phrenic nerve paralysis were still considerable, requiring further efforts for improvement (34). Such data highlights that regionalization efforts hold promise, but require continuous monitoring to ensure the provision of the highest CHS quality possible for patients.

These efforts have not extended to the global community, which is currently developing paradigms for ideal CHS care. Regionalization of CHS care in low- and 
middle-income countries is an extensive independent topic, rooted in robust collaborations with in-country surgeons and policy makers leading the change they believe is needed for their patients. The CHS needs in low- and middleincome countries are different than those in high-income countries. Rheumatic heart disease for instance, has been near-eliminated in high resource settings, yet it is prevalent in 40 million people worldwide, and concentrated in low resource settings (35). The political buy-in, national country roles with regional World Health Organization offices, as well as unique geographic barriers to each country and region, pose additional layers of complexity when discussing regionalization in such settings.

Given that the United Nations 2030 agenda for sustainable development encompasses health and wellbeing through sustainable development goal 3 (SDG 3), with a particular focus on universal health coverage through target 3.8 , it is imperative for congenital heart surgeons to initiate trans-national collaborations to further the 2030 agenda. Target 3.2 and 3.c aim to end newborn and under-5 preventable deaths and increase health financing and healthcare worker deployment and retention in developing countries. This highlights a growing need for surgeons to influence and inform policy-making in their capacity as experts in the field as well as a need for regionalization in low resource settings. These efforts will need to be rooted in a theoretical simulated framework, designed in conjunction with local experts, and piloted to assess impact and inform future best practices.

The tools and methodology developed for regionalization can serve a different purpose in low- and middle-income countries. These countries have limited resources allocated for healthcare and therefore must distribute physical and human resources extremely efficiently. Rather than closing hospitals and focusing care at remaining regional centers as in high-income countries, in low- and middle-income countries the methodology of regionalization can be used to plan where to place centers to best serve the population needs in the most economical and efficient manner. It should also focus on the acuity of the congenital cardiac surgical procedure, anticipated case volume, level of technical skill required to perform the procedure, as well as infrastructure and resources (36).

\section{Key areas of focus for future research}

A clearer understanding of methods to curtail challenges faced by patients in low- and middle-income countries is needed. The full spectrum of CHS requires attention on several levels: timeliness of diagnosis, availability of modes of transportation, respective infrastructure, availability of resources, technical expertise, and the structure of postoperative surveillance. Situational analyses focused on these topics would be important in either the development of national healthcare delivery programs, or the modification of existing paradigms. Furthermore, data regarding the aforementioned areas and desired states should be coupled with a plan aimed at reforming overall health policy and governance structure within each country and region, as well as mechanisms to ensure accountability.

Coupling of these interests have been successfully used previously. It has been shown that further collaboration with political institutions is needed to advance the use of health evidence in policymaking (37). In 2016, the World Society for Pediatric and Congenital Heart Surgery established its plan for international exchange of trainees to enhance international surgical education and treatment missions in addition to a potential system for global certification of its surgeons to facilitate movement of skill to areas of need (38). Such plans are promising and future research efforts can potentially collaborate with global surgical technical experts to ensure that international efforts, such as the one announced by the World Society, are supported with the relevant expertise to set up local, national and regional capacity building mechanisms. Research could also focus on assessing the impact of such interventions and disseminating lessons learned to nearby regions or nearby countries in the same region. For example, the 'Partnership for the Heart' training initiative between Germany and Estonia demonstrated a $28 \%$ reduction in infant mortality secondary to CHD at $50 \%$ the cost incurred if the patients had to leave Estonia to seek care (39).

Multiple other areas of research also will be critical to understanding the impact of regionalization. Studying whether SES and race are impacted adversely by regionalization, a potentially large challenge, are needed. Investigating the potential presence of an inflection point at which surgical volumes exceed the ability of a center to ensure top quality care, especially in low resource settings, where centers may have an excessive number of cases, will be important. The high volume of cases done in some LMIC will potentially facilitate the discovery of such an inflection point-although this will depend on the surrounding infrastructure to be evolved to a point where these systems can be compared to those of a developed country. 


\section{Conclusions}

In high-income countries, regionalization has the potential to reduce mortality and cost. In LMICs, regionalization is a promising model capable of increasing access to timely, affordable and quality CHS services, as advocated for by the Lancet Commission on Global Surgery. Furthermore, centralization in developing countries can leverage lessons learned in the U.S. by virtue of their blank slate. It is not clear whether these efforts, locally in the U.S., or abroad will disparately impact patients of lower SES or those of fragile ethnic/racial groups. Financial incentives must be aligned with efforts to improve care deliveryand this may be a difficult battle given that CHS services provide substantial income to hospital systems, and support other pediatric services. Regionalization of CHS is likely possible within the U.S., and in the developing countries, yet requires a multi-disciplinary effort, national and international commitment, political buy-in as well as continuous research to ensure the principles of medical ethics are preserved, funding is ample and quality of care is maintained at a high standard.

\section{Acknowledgments}

Funding: None.

\section{Footnote}

Provenance and Peer Review: This article was commissioned by the Guest Editors (Dominique Vervoort and Marcelo Cardarelli) for the series "Addressing Global Disparities in Pediatric and Congenital Cardiac Care" published in $A M E$ Surgical fournal. The article has undergone external peer review.

Reporting Checklist: The authors have completed the Narrative Review reporting checklist. Available at https:// asj.amegroups.com/article/view/10.21037/asj-21-70/rc

Peer Review File: Available at https://asj.amegroups.com/ article/view/10.21037/asj-21-70/prf

Conflicts of Interest: All authors have completed the ICMJE uniform disclosure form (available at https://asj.amegroups. com/article/view/10.21037/asj-21-70/coif). The series "Addressing Global Disparities in Pediatric and Congenital Cardiac Care" was commissioned by the editorial office without any funding or sponsorship. The authors have no other conflicts of interest to declare.

Ethical Statement: The authors are accountable for all aspects of the work in ensuring that questions related to the accuracy or integrity of any part of the work are appropriately investigated and resolved.

Open Access Statement: This is an Open Access article distributed in accordance with the Creative Commons Attribution-NonCommercial-NoDerivs 4.0 International License (CC BY-NC-ND 4.0), which permits the noncommercial replication and distribution of the article with the strict proviso that no changes or edits are made and the original work is properly cited (including links to both the formal publication through the relevant DOI and the license). See: https://creativecommons.org/licenses/by-nc-nd/4.0/.

\section{References}

1. Karamlou T, Jacobs ML, Pasquali S, et al. Surgeon and center volume influence on outcomes after arterial switch operation: analysis of the STS Congenital Heart Surgery Database. Ann Thorac Surg 2014;98:904-11.

2. Karamlou T, Johnston DR, Backer CL, et al. Access or excess? Examining the argument for regionalized cardiac care. J Thorac Cardiovasc Surg 2020;160:813-9.

3. Dong GN. Performing well in financial management and quality of care: evidence from hospital process measures for treatment of cardiovascular disease. BMC Health Serv Res 2015;15:45.

4. Welke KF, Pasquali SK, Lin P, et al. Theoretical model for delivery of congenital heart surgery in the United States. Ann Thorac Surg 2021;111:1628-35.

5. Healthy People 2020. Office of Disease Prevention and Health Promotion. US Department of Health and Human Services Phase I Report: Recommendations for the Framework and Format of Healthy People 2020. Section IV. Advisory Committee Findings and Recommendations. [Accessed June 5, 2020]. Available online: https://www. healthypeople.gov/2020/about/advisory/Reports

6. Oster ME, Strickland MJ, Mahle WT. Racial and ethnic disparities in post-operative mortality following congenital heart surgery. J Pediatr 2011;159:222-6.

7. Gonzalez PC, Gauvreau K, Demone JA, et al. Regional racial and ethnic differences in mortality for congenital heart surgery in children may reflect unequal access to care. Pediatr Cardiol 2003;24:103-8. 
8. Peterson JK, Chen Y, Nguyen DV, et al. Current trends in racial, ethnic, and healthcare disparities associated with pediatric cardiac surgery outcomes. Congenit Heart Dis 2017;12:520-32.

9. Karamlou T, Peyvandi S, Federman M, et al. Resolving the Fontan paradox: Addressing socioeconomic and racial disparities in patients with a single ventricle. J Thorac Cardiovasc Surg 2018;155:1727-31.

10. Birkmeyer NJ, Gu N, Baser O, et al. Socioeconomic status and surgical mortality in the elderly. Med Care 2008;46:893-9.

11. Konety SH, Vaughan Sarrazin MS, Rosenthal GE. Patient and hospital differences underlying racial variation in outcomes after coronary artery bypass graft surgery. Circulation 2005;111:1210-6.

12. Schelbert EB, Rosenthal GE, Welke KF, et al. Treatment variation in older black and white patients undergoing aortic valve replacement. Circulation 2005;112:2347-53.

13. Karamlou T, Hawke JL, Zafar F, et al. Widening our Focus: Characterizing Socioeconomic and Racial Disparities in Congenital Heart Disease. Ann Thorac Surg 2022;113:157-65.

14. Woo JL, Anderson BR, Gruenstein D, et al. Minimum travel distance among publicly insured infants with severe congenital heart disease: potential impact of in-state restrictions. Pediatr Cardiol 2019;40:1599-608.

15. Welke KF, Pasquali SK, Lin P, et al. Hospital distribution and patient travel patterns for congenital cardiac surgery in the United States. Ann Thorac Surg 2019;107:574-81.

16. Satyanarayana Rao KH. Informed consent: an ethical obligation or legal compulsion? J Cutan Aesthet Surg 2008;1:33-5.

17. Patel A, Gandhi R. An Ethical Argument for Professional Regulation and Regionalization of Care in Pediatric Cardiology and Cardiac Surgery. Pediatr Cardiol 2020;41:651-3.

18. Saxena A. Congenital cardiac surgery in the less privileged regions of the world. Expert Rev Cardiovasc Ther 2009;7:1621-9.

19. Shukla VV, Bobhate P, Mohanty S, et al. Early outcomes of neonatal cardiac surgery in India. Indian Pediatr 2020;57:129-32.

20. Raj S, Swaminathan S. Early outcomes after cardiac surgery in neonates and infants in India. Indian Pediatr 2020;57:117-8.

21. Khongphatthanayothin A, Layangool T, Sittiwangkul R, et al. Pediatric heart surgery waiting time in Thailand and its effect on mortality: a cooperative study from
Chulalongkorn, Children and Chiang Mai University hospitals. J Med Assoc Thai 2005;88 Suppl 4:S23-9.

22. Okonta KE, Tobin-West CI. Challenges with the establishment of congenital cardiac surgery centers in Nigeria: survey of cardiothoracic surgeons and residents. J Surg Res 2016;202:177-81.

23. Vervoort D, Vinck EE, Tiwari KK, et al. Cardiac surgery and small island states: a bridge too far? Ann Thorac Surg 2021;111:931-6.

24. Edwin F, Entsua-Mensah K, Sereboe LA, et al. Conotruncal heart defect repair in Sub-Saharan Africa: remarkable outcomes despite poor access to treatment. World J Pediatr Congenit Heart Surg 2016;7:592-9.

25. Edwin F, Zühlke L, Farouk H, et al. Status and challenges of care in Africa for adults with congenital heart defects. World J Pediatr Congenit Heart Surg 2017;8:495-501.

26. Edwin F, Elgamal MA, Dorra A, et al. Challenges of caring for functionally single ventricle patients in Africa. World J Pediatr Congenit Heart Surg 2019;10:338-42.

27. Edwin F. Palliation of functionally single ventricle patients in sub-saharan africa-is it justifiable? World J Pediatr Congenit Heart Surg 2019;10:171-3.

28. World Health Organization. Strengthening emergency and essential surgical care and anaesthesia as a component of universal health coverage. [Accessed July 13, 2021]. Available online: https://apps.who.int/gb/ebwha/pdf_files/ WHA68/A68_R15-en.pdf

29. Zheleva B, Atwood JB. The invisible child: childhood heart disease in global health. Lancet 2017;389:16-8.

30. Vervoort D, Cardarelli $M$. The global unmet need of congenital cardiac care: a quantitative analysis of the global burden of disease. Cardiol Young 2020;30:1688-93.

31. Vervoort D, Meuris B, Meyns B, et al. Global cardiac surgery: access to cardiac surgical care around the world. J Thorac Cardiovasc Surg 2020;159:987-996.e6.

32. Vervoort D, Swain JD, Pezzella AT, et al. Cardiac surgery in low- and middle-income countries: a state-of-the-art review. Ann Thorac Surg 2021;111:1394-400.

33. Lundström NR, Berggren H, Björkhem G, et al. Centralization of pediatric heart surgery in Sweden. Pediatr Cardiol 2000;21:353-7.

34. Hirata Y, Hirahara N, Murakami A, et al. Current status of cardiovascular surgery in Japan, 2015 and 2016: a report based on the Japan Cardiovascular Surgery Database. 1-congenital heart surgery. Gen Thorac Cardiovasc Surg 2019;67:731-5.

35. Peters F, Karthikeyan G, Abrams J, et al. Rheumatic heart disease: current status of diagnosis and therapy. Cardiovasc 
Diagn Ther 2020;10:305-15.

36. Iverson KR, Svensson E, Sonderman K, et al.

Decentralization and regionalization of surgical care: $\mathrm{a}$ review of evidence for the optimal distribution of surgical services in low- and middle-income countries. Int J Health Policy Manag 2019;8:521-37.

37. Liverani M, Hawkins B, Parkhurst JO. Political and institutional influences on the use of evidence in public health policy. A systematic review. PLoS One

doi: 10.21037/asj-21-70

Cite this article as: Ghandour HZ, Welke K, Karamlou T. The road to regionalization in congenital heart surgery: a narrative review. AME Surg J 2022;2:27. 2013;8:e77404.

38. Jonas RA. Strengthening international collaboration in congenital heart surgery. World J Pediatr Congenit Heart Surg 2018;9:383-91.

39. Köhler F, Schierbaum C, Konertz W, et al. Partnership for the heart. German-Estonian health project for the treatment of congenital heart defects in Estonia. Health Policy 2005;73:151-9. 